\title{
PRE-OPERATIVE PREPARATION OF A PATIENT WITH DISSEMINATED GIST
}

\author{
Anjana Vasudevan ${ }^{1}$, Ponniah Iyyappan², C. Kaliyappa ${ }^{3}$, Jagdeesh Chandra Bose ${ }^{4}$
}

${ }_{1}^{1}$ Senior Resident, Department of General Surgery, SRMC, Porur, Chennai, Tamilnadu, India.

2 Assistant Professor, Department of General Surgery, SRMC, Porur, Chennai, Tamilnadu, India.

3 Professor, Department of General Surgery, SRMC, Porur, Chennai, Tamilnadu, India.

4 Professor and HOD, Department of General Surgical Oncology, SRMC, Porur, Chennai, Tamilnadu, India.

HOW TO CITE THIS ARTICLE: Vasudevan A, Iyyappan P, Kaliyappa C, et al. Pre-operative preparation of a patient with disseminated GIST. J. Evolution Med. Dent. Sci. 2018;7(42):4589-4590, DOI: 10.14260/jemds/2018/1023

\section{PRESENTATION OF CASE}

Here we present a 68-year-old female, who was diagnosed with GIST. Pre-operatively, the patient underwent a CT abdomen with contrast. This showed multiple deposits over the spleen.

We prepared her for a splenectomy by giving her pneumococcal and meningococcal vaccines. Three weeks later she underwent sleeve gastrectomy with omentectomy and splenectomy. Post-operative period was uneventful. She was started on diet on POD 3 and she tolerated it well.

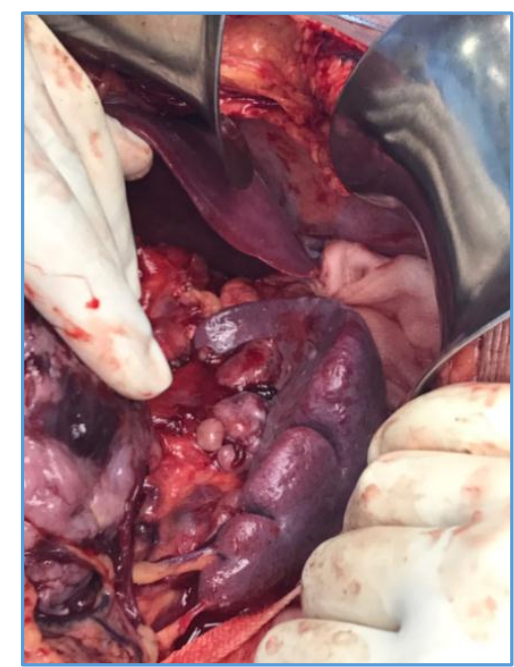

Figure 1. Intraoperative Picture showing Deposits in the Spleen

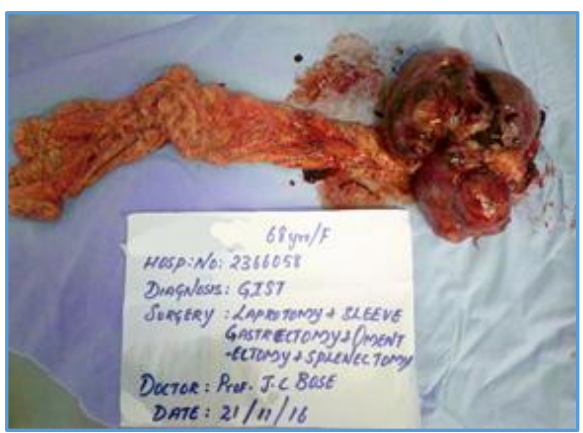

Figure 2. Showing the removed Specimen in toto-a part of the Stomach with the Tumour with Omentum and Spleen

'Financial or Other Competing Interest': None.

Submission 27-08-2018, Peer Review 28-09-2018,

Acceptance 04-10-2018, Published 15-10-2018.

Corresponding Author:

Dr. Anjana Vasudevan,

Sri Ramachandra University, Porur,

Chennai-600018, Tamilnadu, India.

E-mail: dr.anjanavasudevan@hotmail.com

DOI: $10.14260 /$ jemds $/ 2018 / 1023$

\section{(c) (1) $(9)$}

Subsequently, her histopathology reports suggested highgrade spindle cell variant of GIST with CD-117 positivity and Ki67 indexing label of $10 \%$.

\section{CLINICAL DIAGNOSIS}

Disseminated High-Grade Spindle Cell Variant of Gastrointestinal Stromal Tumour.

\section{DIFFERENTIAL DIAGNOSIS}

Schwannomas, Lymphoma, Intra-Abdominal Fibromatosis, Inflammatory Fibroid Polyps, Paragangliomas, Carcinoid Tumours, Sarcomas and Adenocarcinomas.

\section{PATHOLOGICAL DISCUSSION AND DISCUSSION OF MANAGEMENT}

Non-epithelial neoplasms of the gastrointestinal tract that do not have the ultrastructural characteristics of smooth muscle cells are known as GISTs. Mazur and Clark in 1983 were the first to describe GISTs. GISTs can be differentiated from Schwann cells by the lack of the immunohistochemical features characteristic to the Schwann cells. A further characteristic of the GISTs is the strong expression of the KIT protein in immunohistochemistry.

Further studies have revealed the GISTs to be spindle cell, epithelioid or occasionally pleomorphic mesenchymal tumours of the gastrointestinal tract. GIST tumours arise because of gain of function mutations in the KIT proto oncogene. This discovery by Hirota and colleagues in 1998 was important, because it is the KIT proto oncogene which encodes the KIT protein and as previously discussed GISTs strongly expresses the KIT protein. In summary, GISTs can be considered as encompassing intra-abdominal non-epithelial (mesenchymal) tumours that express the KIT protein or have activating mutation in a class III receptor tyrosine kinase gene (KIT or PDGFRA). ${ }^{(1,2)}$

The median age of diagnosis is 65 years. In fact, Gastrointestinal stromal tumours (GIST) are the most common mesenchymal tumours of the Gastrointestinal (GI) tract. They originate from the interstitial cells of Cajal, whose function is to act as pacemaker cells. In majority of the cases, GIST occurs in the stomach (60-70\%). They are also found in the small intestine in about $20-30 \%$ of the patients. Rare occurrence of GIST has also been reported in the abdominal cavity, specifically in the mesentery, omentum and retroperitoneum. GISTs are capable of spreading to the liver and within the abdominal cavity itself. However, bone, lung and lymph nodal metastasis are rare. Metastatic disease at the time of presentation with or without peritoneal seeding indicates a poor prognosis. $(1,3)$

In $75 \%$ of the cases, the primary mutation is found in exon 11, which encodes for the juxtamembrane domain of the protein. This leads to an activation of the receptor regardless 
of the presence of its ligand (Stem cell factor). In $15 \%$ of the cases, mutations are found in Exon 9 of the c-KIT gene encoding the extracellular domain. In another $5 \%$ of the cases, Exons 13 and 17, encoding the kinase domain of the protein are mutated. In approximately $5 \%$ of cases, mutations are found in the homologous gene PDGFRA (platelet-derived growth factor receptor alpha). The remaining $12 \%$ GIST are wild type for both c-KIT and PDGFRA genes. Mutational status of c-KIT has emerged as a major prognostic and predictive factor in patients with GIST.(4)

Other chromosomal abnormalities in GIST exist. Particularly monosomy of chromosomes 14 and 22 as well as deletions of $1 p$ are well known. These abnormal characteristics have been limited to use in pathogenesis and are currently now used as diagnostic characteristic adjuncts.(2) The differential diagnosis for GISTs currently include intra-abdominal fibromatosis, inflammatory fibroid polyp, paragangliomas and metastatic malignant melanoma. ${ }^{(1,4)}$

Diagnosis of GIST is confirmed by a biopsy indicating CD117 positivity. In case of CD-117 negativity, the tumours require further testing with C-KIT mutations and PDGFRA mutations. The size, location and extent of GIST spread can be determined by CT and MRI. The immunohistochemical analysis of PTEN is also a useful tool in prognosis, since as GIST progresses the phosphatase and tensin homologue cannot be observed on chromosome 10. PTEN downregulation is implied in GIST progression.(1)

The established treatment for localised GISTs is a complete surgical resection. However, after complete surgery, the risk of relapse is approximately $40 \%$. The other standard of care in this setting is imatinib. It has been demonstrated that imatinib significantly improves survival in patients with advanced GIST since as an oral, selective and small-molecule tyrosine kinase inhibitor Imatinib targets the Kit protein and the PDGFRA. Furthermore, adjuvant imatinib administered in high-risk patients for 12 months after surgical removal of GIST has been shown to prolong Recurrence-Free Survival (RFS) compared to use of a placebo. Based on these results, imatinib was approved at a daily dose of $400 \mathrm{mg}$ by the US Food and Drug Administration (FDA) and the European Medicine Agency (EMA) in 2008 and 2009, respectively, as adjuvant therapy for high-risk patients following complete surgical resection of GIST.(4,5)

\section{REFERENCES}

[1] De Silva MVC, Reid R. Gastrointestinal stromal tumours (GIST). Orphanet encyclopedia 2005:1-6.

[2] Joensuu H, Kindblom LG. Gastrointestinal stromal tumors - a review. 2017;6470 .

[3] ESMO / European Sarcoma Network Working Group. Gastrointestinal stromal tumors: ESMO Clinical Practice Guidelines for diagnosis, treatment and follow-up. Ann Onco 2012;23(Suppl 7):vii49-55.

[4] Koumarianou A, Economopoulou P, Katsaounis P, et al. Gastrointestinal Stromal Tumors (GIST): a prospective analysis and an update on biomarkers and current treatment concepts. Biomark Cancer 2015;7(Suppl 1):1-7.

[5] Novartis. Gleevec prescribing information. 2008 http://www.accessdata.fda.gov/drugsatfda_docs/labe l/2008/021588s024lbl.pdf 